\title{
Analysis of the "Publicity" of Network Community of Science Communication - Case Studies of Guokr.com and Songshuhui-Association of Science Communicators
}

\author{
Qin Wang ${ }^{1}$ \\ ${ }^{1}$ College of Liberal Arts, Dongguan University of Technology, Dongguan, Guangdong, China \\ Correspondence: Qin Wang, College of Liberal Arts, Dongguan University of Technology, Dongguan, \\ Guangdong, China. E-mail: wangqinjaney@126.com
}

Received: August 11, 2015 Accepted: October 14, 2015 Online Published: October 27, 2015

doi:10.5539/ass.v11n27p1 URL: http://dx.doi.org/10.5539/ass.v11n27p1

\begin{abstract}
Considering public sphere as the research perspective, this paper conducts a study on public sphere of science communication that is set up by scientific social contact media which are represented by Guokr.com and Songshuhui-Association of Science Communicators. Then, the paper makes deep discussion on the "publicity" of network communy of science communication and the crisis it may encounter (namely, exclusiveness and identity landscape of elite communities).
\end{abstract}

Keywords: public sphere, public sphere of science communication, Guokr.com, Songshuhui-Association of Science Communicators, network community, publicity, communities

\section{Proposal of Research Questions}

With advent of scientific revolution once again worldwide, network virtual community which has arisen over the past few years has offered brand-new approaches to scientific communication for network users by virtue of its powerful internet interactive platform. In network virtual community, opinion leaders of network community of science communication are qualified to set up topics of discussion for scientific community and spread scientific knowledge and information to common users of the community. Community members are also entitled to build and share scientific knowledge information, pictures and videos and express their opinions and viewpoints with freedom. They gather together based on common interest and form a social and civic community which takes interest in science and technology affairs. An individual of the community no longer passively and totally accepts all scientific knowledge information of content producers. Instead, he absorbs information in a reflective and critical way. In the public sphere of science communication, an individual of the community is entitled to make independent self-decision on the scientific and technical affairs which he shows interest in and in which he actively takes part. In this way, his subject consciousness gets released to the largest extent. How does the network virtual public space established by scientific social contact media reflect "publicity" of the community? At present, which crises are encountered by "publicity" of network community of science communication? This paper takes Guokr.com and Songshuhui-Association of Science Communicators as a case study and makes profound analysis of the above issues based on the research perspective of public sphere.

\section{Study on Relevant Theories in Public Sphere}

The concept of public sphere was firstly put forward by the German American Hannah Arendt and the German scholar Jürgen Habermas. In 1958, Hannah Arendt, for the first time, put forward the concept of public sphere. In her book "The Human Condition", she mentioned, "the word 'public' means two phenomena that are closely connected but are not totally identical. Any phenomenon that is displayed to the public sphere has to be seen and heard by each person and has the maximum publicity." (Note 1) In 1961, Habermas conducted a profound research on evolution and transition of public sphere. According to his theory, public sphere and life world are two closely connected concepts. He pointed out in his theoretical works "Structural Transformation of the Public Sphere", "if a communication action does not take its root in a backdrop of life world which offers large-scale background consensus, such a risk will lead to the result that language use inclined to mutual understanding does not fulfill social integration. At the very beginning, a communication action is set in a shared atmosphere that has no doubt. In the meantime, it obtains nutrient from those resources which it has got familiar with quite earlier." 
(Note 2) In this sense, he believes that life world is a source for all elements in the public sphere and constitutes understanding of the public in daily living practice in the public sphere and a context in which the public reach a consensus based on their understanding. The following three factors are required to constitute a public sphere. Firstly, it is the public who have critical consciousness and have independent personality and critical spirit and who are able to participate in any discussion on a rational basis regarding problems of public interest. Secondly, it is the media that can communicate freely and fully. Finally, it is to form public opinions. In a public sphere, the public may make discussion with freedom and communicate fully concerning public topics and public affairs and reach a consensus on a rational and critical basis, which will form public opinions.

\section{Science Communication from the Perspective of Public Sphere: Construction of Public Sphere with Science Communication}

The word "science communication" was, at the earliest, used by the British scholar J.D Bernal in his book "The Social Function of Science". It has undergone communication at three phases, which are respectively central broadcasting model, deficit model and dialog model (also termed as democracy model). These three types of science communication models correspond to the traditional period of popularization of science, the traditional period of public understanding of science and the traditional period of "reflective" science communication. (Note 3) After network communication springs up, especially when social contact media become an important usage by network users, due to interactivity and openness of the media per se, quite a large quantity of researches hold the view that science communication in China has entered the period of "reflective" science communication. (Note 4) The "reflective" science communication is characterized by diversification of science communication subject and object, focus on its attitude towards the public, the right to speak of the public and participation in science and technology affairs by the public. The theory of "the fourth subject" further poses that the dialog model lays more stress on expression of "household interest". (Note 5) Therefore, discussion on establishment of the "reflective" science communication model and construction of the public sphere of science communication may not avoid the following several issues: whether the public participation in science and technology affairs is realized to a maximum extent, whether the subject consciousness of a network individual is given full play, tolerance and acceptance of different opinions in the public sphere of science communication by the public, the awakening capacity of an individual's subject consciousness, his public spirit and his rationally critical spirit as well as whether self-construction and self-correction capacity of the knowledge information is adequately fulfilled. Then, in the process of constructing the public sphere of science communication, how to realize equal "dialogue" and "mutual communication" of knowledge between opinion leaders and community individuals in the network community of science communication; how the subject consciousness centered by "reflectiveness" and critical spirit "germinates" in the virtual public space. This paper will make an analysis and conduct research on all the issues by taking such scientific and social contact network as Guokr.com and Songshuhui-Association of Science Communicators as examples to reflect dialectically on these issues.

\section{An Analysis of the "Publicity" of Network Community of Science Communication - A Case Study of Guokr.com and Songshuhui-Association of Science Communicators}

\subsection{Equal "Dialogue" and "Mutual Communication" of Information Exist between Opinion Leaders and Community Individuals}

In the process of constructing the public sphere of science communication, analysis of "publicity" of the network community should, first of all, focus on mutual communication of knowledge information between opinion leaders and common individuals. That is to realize publicity of the network space in the process of equal "dialogue". In his Hermeneutics theory, the German Philosopher Hans-Georg Gadamer points out, "understanding is made possible in dialogue", which means that "without dialogue, there won't be any possibility to reach a consensus". Habermas puts forward the "communicative rationality". He points out, communicative rationality is two-dimensional which involves the dialogical relationship between different speakers. The traditional concept of rationality is manifested through our paradigm of knowledge in an object, whereas communicative rationality is expressed by the paradigm that the subjects are mutually understood. These subjects are able to speak and behave and live in understanding of a world of non self-centration. It is rationality of a life world and concentrates on inter-subjectivity with reliable propositions. People under different contexts, through social contact, achieve mutual respect and avoid misunderstanding and misinterpretation so as to fulfill "sympathetic understanding" and certain kind of real "consensus". The well-known French sociologist Pierre Bourdieu poses, "when people compete in their own field domain, they are not attempting to overwhelm each other, but are, instead, taking into consideration mutual understanding and innovation." As a network medium, Songshuhui-Association of Science Communicators follows the idea of "diversity, equality, openness and interactivity". It not only "popularizes" scientific information and knowledge up to date to the public who 
are "a fool" to science, as a result of which the public is respected and is responded patiently. What's more, the platform of this association corrects even professional disseminators. In the meantime, in the process of an equal dialogue, the disseminators have the opportunity to know more about their audience and communicate with and know about each other in the dialogue, which avoids science communication from deviating purport and interest of network community users. Chen Chao, a member of Songshuhui-Association of Science Communicators, poses that this association, to a certain extent, has avoided the boundedness of communication with a small circle of people. The association gives members and users of the community an opportunity to publish articles which are directly checked by a mass of readers and receive replies from members of the community among which there are really penetrating opinions and nitpick for correctness of science. (Note 6) In addition, Guokr.com has also made practical attempts in this regard. In the following, we are going to take as an example a talent with an internet ID " $\mathrm{Zi} \mathrm{Yu"} \mathrm{in} \mathrm{Guokr.com} \mathrm{to} \mathrm{analyze} \mathrm{how} \mathrm{Guokr} \mathrm{talents} \mathrm{as} \mathrm{opinion} \mathrm{leaders} \mathrm{and} \mathrm{the} \mathrm{common} \mathrm{users} \mathrm{of}$ Guokr community attain equal dialogue in a virtual public space. In the real life, "Zi Yu" talent is a mater in phytoecology, while in Guokr.com, he is not merely a Guokr talent, but also takes charge of the two small groups of "natural control" and "stargazer". "Zi Yu" talent ever said, "he even went to Mount Emei for seven times for a forest quadrat and was ever stationed in the forest where the film 'The Twilight Saga' was shot to examine the plantlets of needle-leaved trees and measure their photosynthetic intake." Such a talent who possesses such abundant professional and practical experience often introduces, in Guokr community, the novel biological creatures he has ever encountered when travelling around the world, such as, the protogenetic orchid observed in Amazon, the rufous-collared sparrow he ever came across in Spain, the white bears he say in the Arctic Pole and so on. Besides, he also introduces to Guokr community members all kinds of lovely animals and explains the miraculous constellation meteor shower. His vivid and interesting explanation full of wisdom gains support from other community members. In his discussion in the virtual public space of Guokr community, "Zi Yu" who possesses the authority of discourse also pays attention to exchange of thoughts of information and emotional interaction. His equal "dialogue" with the common users makes interpretation to the publicity of the network virtual space at the practical level.

\subsection{Construction of "Interest-Related" Social and Civil Community}

In his theoretical book "Human Emotions: A Sociological Theory", Jonathan H. Turner points out, emotion (including interest) originates from the framework of the social structure and the sociological theory should explain which emotion (including interest) is activated, the intensity level of the emotion activated (including interest), the directing object and what influences this kind of emotion (including interest) will bring to individuals and the social structure. (Note 7) This viewpoint of Jonathan H. Turner is of guidance significance to this paper in its research of the "publicity" of public sphere and network community of science communication. The contemporary society is in the face of transition from a traditional society to a modern society, which is not merely transition in terms of system and structure, but is also transition of the psychological structure of an individual. The tripping point in transition of individual psychological structure triggering transition of social contact in the network community of science communication is exactly the interest point that hides deeply in the heart of individuals of the communities. In the following, we are going to take as an example Guokr to analyze how, in the process of constructing the public sphere of science community, the communities of science community realize free cohesion based on "interest-related destiny" in the network virtual public space.

In order to strengthen cohesive force of the network communities, Guokr.com has set up "group-based" module. Establishment of group-based module aims to gather Guokr.com net friends and to form the culture of communities with common interest. "Group-based" modules are set up by users of Guokr community who generate their content. Guokr users also have the right to attract other users to take part in the discussion. The quantity, quality and degree of popularity of posts at the bottom of each group identify vitality and attraction of this group. Sponsors and participants of a group gradually define the scope and boundary of topics for discussion in the process of discussion so as to form and comply with culture of Guokr communities. Currently, what are relatively popular groups in Guokr.com include Greek low laughing points, foodie institute, challenge of procrastination, universal gravitation and so on. Therefore, the "group-based" module of Guokr.com forms different scales of scientific social circles based on different interests by virtue of classification of different groups within the community.

Based on the above statement made by Habermas in his theoretical works "Structural Transformation of the Public Sphere" that public sphere and life world are two closely connected concepts, it is necessary to apply abstruse and difficult scientific knowledge into daily living practice and routinize it in the process of constructing the public sphere of science communication. This aims to make "use science" and "play science" become common behaviors of members of scientific social contact network community. Then, the scientific 
knowledge is matched with language full of wit and humor. In order to further intensify the motive of members of scientific social contact network community to probe into scientific knowledge, it is necessary to seek for "interest points" in practical application of science and in public discussion. For instance, all the following topics in the hot posts of the groups at the end of July 2014 are full of wisdom and interest and are close to daily life, such as, "Is the traditional method of temperature falling in a car reliable", "Are golden rice and yellow banana meaningful", "Is it a must to drink hot water in the hottest days in summer to excrete chill" and so on. These topics have been discussed with high enthusiasm in the Kuokr communities. There are also some other topics which are close to daily life, such as, "Will the system start its automatic alarm if one enters his password in an inverse order on an ATM", "What will the world be if there is no mosquito" and so on. Users of the Kuokr.com make spontaneous discussion on these issues and attempt to resolve these issues by means of looking up document, doing hands-on experiment and reasoning and proving. It is worth mentioning that communities members with different disciplinary backgrounds and different professional and practical experiences gather together freely in a virtual public space and, guided by problems, make fierce discussion on topics of common interest. They together resolve problems through collision of thoughts of information, experimental practice and logic inference.

Moreover, implication of "interest-related" is not merely restricted to aforementioned "interest" (namely, internal motive to probe into scientific knowledge and thirst for knowledge), but also, meanwhile, contains mutual tickling and "amusing" science in a sportive way, seeking for "interest points", and searching for the sense of belonging to the communities through collective onlooking of network community and carnival within a circle. For example, in April 2012, a cross-talk spread out on the internet about "dihydrogen monoxide". This cross-talk detailed a lot of "truths" about "dihydrogen monoxide" that were unbeknown before and warned the public to be noticed of dangers of "dihydrogen monoxide". These include, but not limited to, "it is the major ingredient of acid rain; it has facilitating effect on soil erosion; it boosts greenhouse effect; it is the cause for corrosion; too much intake of dihydrogen monoxide might lead to discomforts to different extents; if the skin gets contact with its solid form for long, it may result in serious damage of tissue; people addicted to dihydrogen monoxide will die without it for 168 hours", and so on. However, as a matter of fact, all these so-called dangers are nothing more than description of the functions of "dihydrogen monoxide" (namely, water) in sensational mood. Net friends of Guokr.com forwarded this cross-talk to the group of "Greek low laughing points" which attracted a large number of Guokr.com community users. Quite a lot of Guokr community users, one after another, left a message, such as, "Please forward it for those you care and let more know about it." Moreover, some users even sportively said, "I feel as if it is an omen of doomsday. Yes, it must be"; "It should mean one is drowned by saying that one die of too much intake of dihydrogen monoxide, and are there a hundred million people?" and so on. These sportive discussion on the above cross-talk by the Guokr community is the formation basis of "interest-related" scientific and social community. That is to say, the community search for "interest points" or "banter points" in hot topics based on interest, which displays the community carnival "landscape" of "amusing to death".

\section{3 "Germination" of the Subject Consciousness Centered by "Reflectivity" and "Critical Spirit"}

Habermas ever poses "It is better to describe public sphere as a contact network about content and viewpoints, namely, opinions. Therein, the flow of contact is screened and synthesized in an ad hoc way and, hence, becomes public views or opinions formed according to collection of particular topics. Just as the whole life world, public sphere also fulfills re-production by means of contact action. Of course, for this action, it is enough to grasp the natural language. It is suitable for the general understandability possessed by daily contract language." (Note 8) Habermas understands public sphere as a kind of social space that is formed in action of social contract. The bi-directional inter-subjectivity achieved owing to encounter in public space depends on recognition of contact freedom. Thus, public sphere provides the citizens with a space of contact between the nation and the civil society, while the citizens are entitled to fully express their own subjectivity in the process of equal contact at the buffer area of public sphere. In the public sphere of science communication, the public are able to obtain "independence" space in the process of active participation in science and technology affairs. Accordingly, their subjectivity gets liberated to a large extent. The public possess the subject consciousness with "reflective" and critical spirit and acquire the ability of independence and self-determination. For example, Guokr community users acquire self-subjectivity cognition by means of cracking and distinguishing all kinds of rumors in network community and in real life and obtain the ability of independence, self-determination and critical cognition. The special content area of Guokr.com sets up the module of "testing your 'rumor resistance' to help community members to realize self-cognition. Guokr users can draw randomly ten subjects to test their ability to crack pseudoscientific speeches in network community and in reali life. The network back-end gives a mark according 
to the options chosen by community members and finally judges the grade of rumor resistance by community users. Rumor resistance includes three grades, namely, "drop-hammer vehicle grade" which is to make all rumors no place to hide, "impact brick grade" with sharp eyesight and "brick grade" which requires more efforts of the users. Setting of the module of "testing your "rumor resistance", to a large extent, activates critical subject consciousness of community users in distinguishing pseudoscientific speeches and helps enhance community users' ability of self-awakening, self-consciousness, self-judgment and self-determination in the public sphere of science communication. "Reflective" and critical subject consciousness "germinates" in social contact in the public space of science communication.

\section{Crisis Encountered by "Publicity" of Network Community of Science Communication: Exclusiveness and Identity Landscape of Elite Communities}

An integrated marketing project conducted by BMW in 2011 describes users of Guokr.com as such, "Audience of Guokr.com embrace urban young people with good educational background, scientific common sense and vocational distribution. Its active users include some middle-senior managers from science and technology enterprises who gain positive popularity and brand reputation in mass media and active blog circles and who are able to influence the group of opinion leaders. Off-site fans (such as, fans of its official micro-blog) of Guokr.com are mainly young people who yearn for science and technology and are passionate with life and who show profound interest and hold particular opinions in scientific creation and joy of life. These youngsters have positive participation enthusiasm and communicative force both in online and off-line activities organized by Guokr.com." (Note 9) The above description indicates that followers of Guokr.com conform to certain categorization characteristics, namely, higher educational level, having high authority of discourse in certain domain and so on. Just as Guokr.com, Songshuhui-Association of Science Communicators also exhibits similar categorization characteristics and gradually manifests the exclusive restriction of internal communication within the community group with higher level of educational background and vocational background. This is just as Bath, a Norwegian anthropologist, says in his theoretical works "Ethnic groups and boundaries" that, "A group restricts its "boundary' to exclude others by emphasizing its particular cultural feature." A virtual group which, in itself, is established on the basis of information exchange and has no actual connection, is all the more so. Therefore, after a "label" of categorization defines the major characteristics of a group, the group will naturally generate exclusiveness, namely, "invisible restriction" to those without the characteristics of the group itself. Thus, it can be seen that social contact of users of Guokr.com and Songshuhui-Association of Science Communicators and social contact of its fans group (namely, followers of Guokr.com and Songshuhui-Association of Science Communicators), to a large extent, is only restricted within the group of scientific elites who have higher authority of discourse. Nonetheless, a large majority of and more ordinary people are excluded from the group of fans as they do not possess categorization characteristics, with lower educational qualification, inferior information influences and communicative forces and greater ages.

Another crisis encountered by "publicity" of community is the identity landscape of the community group. As mentioned earlier, the community groups of Guokr.com and Songshuhui-Association of Science Communicators are characterized by categorization and exclusiveness. Moreover, if the tendency of the characterized groups moves forward, it might become a symbolic mark of certain identity, and, furthermore, display identity landscape. It was ever reported by "JiangHuai Morning News" that, "Participation in activities held by Songshuhui-Association of Science Communicators' has been a fashion for youngsters in Beijing and Shanghai." In the meantime, it was also reported that among users of this association, the number of people with a disciplinary background of humanities even exceeds the number of people with a background of science and engineering. What's more, quite a large amount of users label and connect themselves with such obviously urban petty bourgeoisie features as "travel", "film", "music" and even "independent film" and "independent music", etc. (Note 10) However, excessive emphasis on the identity characteristics of urban petty bourgeoisie and on the group characteristics of elite culture may result in presentation of the identity landscape of community groups. Just as Welz and Best define that "Landscape means a new people show a performance, while a large majority of appreciate the performance silently", presentation of the identity landscape of community groups also verifies what is stated by Ludwig Feuerbach in his works "The Essence of Christianity", namely, "symbol prevails over material object, transcript prevails over the original manuscript, representation prevails over reality and phenomenon prevails over nature". When presentation of the identity landscape of the urban petty bourgeoisie group and the elite community group becomes a symbolic sign without the aim of science communication with substantive significance, this, to a certain extent, may lead to exclusive social contact of the elite community group. Instead, the social contact is only restricted to exchange and communication of information within the elite community group. This will also, to certain extent, result in deficiency of the publicity of science 
communication community.

\section{Concluding Remarks}

Scientific social contact media, represented by Guokr.com and Songshuhui-Association of Science Communicators offer a platform of exchange and communication of scientific information and knowledge for network community individuals in the process of science communication. Ranging from opinion leaders of network community of science communication to common network users, scientific social contact media have made it possible for network community individuals to actively take part in science and technology affairs and give full play to their subject consciousness, for the public to tolerate different opinions of the public sphere of science communication and reasonably and critically absorb scientific information as well as for self-construction of knowledge and information. With public sphere as the research perspective, this paper has made analysis and discussion on the "publicity" of network community of science communication and has also made analysis and elaboration on the crises it might encounter (namely, exclusiveness and identity landscape of the elite community group). It is a direction for the research and discussion in the future how to use the analytical method of social network to make quantitative analysis of network community contact of scientific communication.

\section{References}

Arendt, H. (1998). The Human Condition (2nd ed.). Chicago. The University of Chicago Press.

Cheng, M. (2012). The Socialization Characteristics of Network Communication and Establishment of Network Folk Customs. Southeast Communication, (9).

Dong, X. (2012). Brief Analysis of Science Communication at an Era of New Media. News World, (4).

Habermas, J. (1999, January). Structural Transformation of the Public Sphere (1st ed., translated by Cao, Weidong, et al.) Academia Press.

Habermas, J. (2003). Between Facts and Norms: Contributions to a Discourse Theory of Law and Democracy, (p. 446, translated by Tong, Shijun). Beijing: SDX Joint Publishing Company.

Li, Y., \& Yue, K. (2011). Thoughts on Micro Blog from Founding of Guokr.com. Journalism \& Communication, (6).

Liu, H. (2011). "The Fourth Subject" in the System of Science Communication. Impact of Science on Society, (4).

Study on Motives and Use Behaviors of Guokr.com Users. Retrieved from http://www.sojump.com/jq/3140439. aspx

Su, B. (2009). The Status quo of Science Popularization Website in China and Study on Countermeasures. Hefei: University of Science and Technology of China.

Turner, J. H. (2009). Human Emotions: A Sociological Theory (translated by Sun, Juncai \& Wen, Jun). Oriental Publishing.

Wu, J. (2010). Communication Elements of Songshuhui-Association of Science Communicators and Research on Its Communication Patterns. Hefei: University of Science and Technology of China.

Yang, P., \& Shi, D. (2011). Game Playing between Fact and Fiction: Mechanism of Science Communication of Microblog Space - A Case Study of "Rumor Pulverizer". Journalism Quarterly, (4).

\section{Notes}

Note 1. Hannah Arendt. The Human Condition (second edition) Chicago. The University of Chicago Press, 1998

Note 2. [Germany] Jürgen Habermas. Structural Transformation of the Public Sphere (1 $1^{\text {st }}$ ed.), translated by Cao, Weidong, et al. Academia Press, 1999, January.

Note 3. Liu, Huajie. Three Types of Models and Three Phases of Science Communication. Science Popularization, 2009(2).

Note 4. Dong, Xue. Brief Analysis of Science Communication at an Era of New Media. News World, 2012(4).

Note 5. Liu, Huajie. "The Fourth Subject" in the System of Science Communication. Impact of Science on Society, 2011(4).

Note 6. Chen, Chao (Liangzi Xiongmao). After Scientific Training, Another Road outside Scientific Research, 
2011-05-02.

Note 7. Jonathan H. Turner. Human Emotions: A Sociological Theory, translated by Sun, Juncai \& Wen, Jun. Oriental Publishing, 2009.

Note 8. [Germany] Jürgen Habermas. Between Facts and Norms: Contributions to a Discourse Theory of Law and Democracy, translated by Tong, Shijun. Beijing: SDX Joint Publishing Company, 2003, p.446.

Note 9. A Case Study of Integrated Marketing --- JOY Gene of An Automobile. Guokr.com, 2011.

Note 10. http://www.douban.com/group/topic/3070377/, a "required" website for popularization of science by all the people.

\section{Copyrights}

Copyright for this article is retained by the author (s), with first publication rights granted to the journal.

This is an open-access article distributed under the terms and conditions of the Creative Commons Attribution license (http://creativecommons.org/licenses/by/3.0/). 